\title{
Fractional integral problems for fractional differential equations via Caputo derivative
}

\author{
Jessada Tariboon ${ }^{1 *}$, Sotiris K Ntouyas² and Weerawat Sudsutad
}

${ }^{\text {*Correspondence: }}$

jessadat@kmutnb.ac.th

${ }^{1}$ Nonlinear Dynamic Analysis

Research Center, Department of

Mathematics, Faculty of Applied

Science, King Mongkut's University

of Technology North Bangkok,

Bangkok, Thailand

Full list of author information is

available at the end of the article

\section{照 Springer}

\begin{abstract}
In this paper, we study the existence and uniqueness of solutions for fractional boundary value problems involving nonlocal fractional integral boundary conditions, by means of standard fixed point theorems. Some illustrative examples are also presented.
\end{abstract} MSC: 26A33; 34A08; 34B15

Keywords: fractional differential equations; nonlocal boundary conditions; fixed point theorems

\section{Introduction}

Differential equations with fractional order have recently proved to be valuable tools for the description of hereditary properties of various materials and systems. Many phenomena in engineering, physics, continuum mechanics, signal processing, electro-magnetics, economics, and science describes efficiently by fractional order differential equations. For a reader interested in the systematic development of the topic, we refer the books [1-5]. Many researchers have studied the existence theory for nonlinear fractional differential equations with a variety of boundary conditions; for instance, see the papers [6-21], and the references therein.

In this paper, we study the existence and uniqueness of solutions for the following boundary value problem for the fractional differential equation with nonlocal fractional integral boundary conditions

$$
\left\{\begin{array}{l}
{ }^{c} D^{q} u(t)=f(t, u(t)), \quad 1<q \leq 2,0<t<T \\
\sum_{i=1}^{m} \theta_{i} I^{\alpha_{i}} u(T)=\omega, \\
\sum_{j=1}^{n} \lambda_{j} I^{\beta_{j}} u\left(\eta_{j}\right)=\sum_{k=1}^{l} \mu_{k}\left(I^{\gamma_{k}} u(T)-I^{\gamma_{k}} u\left(\xi_{k}\right)\right)
\end{array}\right.
$$

where ${ }^{c} D^{q}$ denotes the Caputo fractional derivative of order $q, f:[0, T] \times \mathbb{R} \rightarrow \mathbb{R}$ is a continuous function, $\eta_{j}, \xi_{k} \in(0, T), \theta_{i}, \lambda_{j}, \mu_{k} \in \mathbb{R}$, for all $i=1,2, \ldots, m, j=1,2, \ldots, n, k=$ $1,2, \ldots, l, \omega \in \mathbb{R}$, and $I^{\phi}$ is the Riemann-Liouville fractional integral of order $\phi>0(\phi=$ $\left.\alpha_{i}, \beta_{j}, \gamma_{k}, i=1,2, \ldots, m, j=1,2, \ldots, n, k=1,2, \ldots, l\right)$.

The significance of studying problem (1.1) is that the boundary conditions are very general and include many conditions as special cases. In particular, if $\alpha_{i}=\beta_{j}=\gamma_{k}=1$, for all $i=1,2, \ldots, m, j=1,2, \ldots, n, k=1,2, \ldots, l$ then the boundary conditions reduce to

$$
\left\{\begin{array}{l}
\left(\theta_{1}+\theta_{2}+\cdots+\theta_{m}\right) \int_{0}^{T} u(s) d s=\omega \\
\lambda_{1} \int_{0}^{\eta_{1}} u(s) d s+\cdots+\lambda_{n} \int_{0}^{\eta_{n}} u(s) d s=\mu_{1} \int_{\xi_{1}}^{T} u(s) d s+\cdots+\mu_{l} \int_{\xi_{l}}^{T} u(s) d s .
\end{array}\right.
$$

C 2014 Tariboon et al.; licensee Springer. This is an Open Access article distributed under the terms of the Creative Commons Attribution License (http://creativecommons.org/licenses/by/2.0), which permits unrestricted use, distribution, and reproduction in any medium, provided the original work is properly cited. 
Note that the condition (1.2) does not contain the values of the unknown function $u$ at the left side and right side of the boundary points $t=0$ and $t=T$, respectively.

We develop some existence and uniqueness results for the boundary value problem (1.1) by using standard techniques from fixed point theory. The paper is organized as follows: in Section 2, we recall some preliminary facts that we need in the sequel and Section 3 contains our main results. Finally, Section 4 provides some examples for the illustration of the main results.

\section{Preliminaries}

In this section, we introduce some notations and definitions of fractional calculus [2, 3] and present preliminary results needed in our proofs later.

Definition 2.1 For an at least $n$-times differentiable function $g:[0, \infty) \rightarrow \mathbb{R}$, the Caputo derivative of fractional order $q$ is defined as

$$
{ }^{c} D^{q} g(t)=\frac{1}{\Gamma(n-q)} \int_{0}^{t}(t-s)^{n-q-1} g^{(n)}(s) d s, \quad n-1<q<n, n=[q]+1 \text {, }
$$

where $[q]$ denotes the integer part of the real number $q$.

Definition 2.2 The Riemann-Liouville fractional integral of order $q$ is defined as

$$
I^{q} g(t)=\frac{1}{\Gamma(q)} \int_{0}^{t} \frac{g(s)}{(t-s)^{1-q}} d s, \quad q>0
$$

provided the integral exists.

Lemma 2.1 For $q>0$, the general solution of the fractional differential equation ${ }^{c} D^{q} u(t)=$ 0 is given by

$$
u(t)=c_{0}+c_{1} t+\cdots+c_{n-1} t^{n-1}
$$

where $c_{i} \in \mathbb{R}, i=1,2, \ldots, n-1(n=[q]+1)$.

In view of Lemma 2.1, it follows that

$$
I^{q c} D^{q} u(t)=u(t)+c_{0}+c_{1} t+\cdots+c_{n-1} t^{n-1}
$$

for some $c_{i} \in \mathbb{R}, i=1,2, \ldots, n-1(n=[q]+1)$.

For convenience we set

$$
\begin{aligned}
& \Omega_{1}=\sum_{i=1}^{m} \theta_{i} \frac{T^{\alpha_{i}+1}}{\Gamma\left(\alpha_{i}+2\right)}, \quad \Omega_{2}=\sum_{i=1}^{m} \theta_{i} \frac{T^{\alpha_{i}}}{\Gamma\left(\alpha_{i}+1\right)}, \\
& \Omega_{3}=\sum_{j=1}^{n} \lambda_{j} \frac{\eta_{j}^{\beta_{j+1}}}{\Gamma\left(\beta_{j}+2\right)}, \quad \Omega_{4}=\sum_{j=1}^{n} \lambda_{j} \frac{\eta_{j}^{\beta_{j}}}{\Gamma\left(\beta_{j}+1\right)}, \\
& \Omega_{5}=\sum_{k=1}^{l} \mu_{k} \frac{T^{\gamma_{k}+1}-\xi_{k}^{\gamma_{k}+1}}{\Gamma\left(\gamma_{k}+2\right)}, \quad \Omega_{6}=\sum_{k=1}^{l} \mu_{k} \frac{T^{\gamma_{k}}-\xi_{k}^{\gamma_{k}}}{\Gamma\left(\gamma_{k}+1\right)}
\end{aligned}
$$


and

$$
\Delta=\Omega_{1}\left(\Omega_{6}-\Omega_{4}\right)-\Omega_{2}\left(\Omega_{5}-\Omega_{3}\right)
$$

Lemma 2.2 Let $\Delta \neq 0,1<q \leq 2, \alpha_{i}, \beta_{j}, \gamma_{k}>0, \eta_{j}, \xi_{k} \in(0, T)$ for $i=1,2, \ldots, m, j=1,2, \ldots, n$, $k=1,2, \ldots, l$ and $h \in C([0, T], \mathbb{R})$. Then the problem

$$
\begin{aligned}
& { }^{c} D^{q} u(t)=h(t), \quad t \in(0, T), \\
& \sum_{i=1}^{m} \theta_{i} I^{\alpha_{i}} u(T)=\omega, \\
& \sum_{j=1}^{n} \lambda_{j} I^{\beta_{j}} u\left(\eta_{j}\right)=\sum_{k=1}^{l} \mu_{k}\left(I^{\gamma_{k}} u(T)-I^{\gamma_{k}} u\left(\xi_{k}\right)\right),
\end{aligned}
$$

has a unique solution given by

$$
\begin{aligned}
u(t)= & I^{q} h(t)+\frac{\left(\Omega_{6}-\Omega_{4}\right) t-\left(\Omega_{5}-\Omega_{3}\right)}{\Delta}\left(\omega-\sum_{i=1}^{m} \theta_{i} I^{\alpha_{i}+q} h(T)\right) \\
& +\frac{\Omega_{1}-\Omega_{2} t}{\Delta}\left(\sum_{j=1}^{n} \lambda_{j} I^{\beta_{j}+q} h\left(\eta_{j}\right)-\sum_{k=1}^{l} \mu_{k}\left(I^{\gamma_{k}+q} h(T)-I^{\gamma_{k}+q} h\left(\xi_{k}\right)\right)\right) .
\end{aligned}
$$

Proof Using Lemma 2.1, (2.4) can be expressed as an equivalent integral equation,

$$
u(t)=I^{q} h(t)+c_{1} t+c_{2} .
$$

Taking the Riemann-Liouville fractional integral of order $p>0$ for (2.7), we have

$$
I^{p} u(t)=I^{p+q} h(t)+c_{1} \frac{t^{p+1}}{\Gamma(p+2)}+c_{2} \frac{t^{p}}{\Gamma(p+1)} .
$$

From the first condition of (2.5) and (2.8) with $p=\alpha_{i}$, it follows that

$$
c_{1} \sum_{i=1}^{m} \theta_{i} \frac{T^{\alpha_{i}+1}}{\Gamma\left(\alpha_{i}+2\right)}+c_{2} \sum_{i=1}^{m} \theta_{i} \frac{T^{\alpha_{i}}}{\Gamma\left(\alpha_{i}+1\right)}=\omega-\sum_{i=1}^{m} \theta_{i} I^{\alpha_{i}+q} h(T) .
$$

According to the above process, the second condition of (2.5) and (2.8) with $p=\beta_{j}$ and $p=\gamma_{k}$ imply that

$$
\begin{aligned}
& c_{1}\left(\sum_{k=1}^{l} \mu_{k} \frac{T^{\gamma_{k}+1}-\xi_{k}^{\gamma_{k}+1}}{\Gamma\left(\gamma_{k}+2\right)}-\sum_{j=1}^{n} \lambda_{j} \frac{\eta_{j}^{\beta_{j}+1}}{\Gamma\left(\beta_{j}+2\right)}\right) \\
& \quad+c_{2}\left(\sum_{k=1}^{l} \mu_{k} \frac{T^{\gamma_{k}}-\xi_{k}^{\gamma_{k}}}{\Gamma\left(\gamma_{k}+1\right)}-\sum_{j=1}^{n} \lambda_{j} \frac{\eta_{j}^{\beta_{j}}}{\Gamma\left(\beta_{j}+1\right)}\right) \\
& =\sum_{j=1}^{n} \lambda_{j} I^{\beta_{j}+q} h\left(\eta_{j}\right)-\sum_{k=1}^{l} \mu_{k}\left(I^{\gamma_{k}+q} h(T)-I^{\gamma_{k}+q} h\left(\xi_{k}\right)\right) .
\end{aligned}
$$


Solving the system of linear equations for constants $c_{1}, c_{2}$, we have

$$
\begin{aligned}
c_{1}= & \frac{\Omega_{6}-\Omega_{4}}{\Delta}\left(\omega-\sum_{i=1}^{m} \theta_{i} I^{\alpha_{i}+q} h(T)\right) \\
& -\frac{\Omega_{2}}{\Delta}\left(\sum_{j=1}^{n} \lambda_{j} I^{\beta_{j}+q} h\left(\eta_{j}\right)-\sum_{k=1}^{l} \mu_{k}\left(I^{\gamma_{k}+q} h(T)-I^{\gamma_{k}+q} h\left(\xi_{k}\right)\right)\right), \\
c_{2}= & -\frac{\Omega_{5}-\Omega_{3}}{\Delta}\left(\omega-\sum_{i=1}^{m} \lambda_{i} I^{\alpha_{i}+q} h(T)\right) \\
& +\frac{\Omega_{1}}{\Delta}\left(\sum_{j=1}^{n} \lambda_{j} I^{\beta_{j}+q} h\left(\eta_{j}\right)-\sum_{k=1}^{l} \mu_{k}\left(I^{\gamma_{k}+q} h(T)-I^{\gamma_{k}+q} h\left(\xi_{k}\right)\right)\right) .
\end{aligned}
$$

Substituting constants $c_{1}$ and $c_{2}$ into (2.7), we obtain (2.6), as required.

\section{Main results}

Let $\mathcal{C}=C([0, T], \mathbb{R})$ denote the Banach space of all continuous functions from $[0, T]$ to $\mathbb{R}$ endowed with the norm defined by $\|u\|=\sup _{t \in[0, T]}|u(t)|$. Throughout this paper, for convenience, the expression $I^{x} f(s, u(s))(y)$ means

$$
I^{x} f(s, u(s))(y)=\frac{1}{\Gamma(x)} \int_{0}^{y}(t-s)^{x-1} f(s, u(s)) d s \quad \text { for } t \in[0, T]
$$

where $x \in\left\{q, \alpha_{i}+q, \beta_{j}+q, \gamma_{k}+q\right\}$ and $y \in\left\{t, T, \eta_{j}, \xi_{k}\right\}, i=1,2, \ldots, m, j=1,2, \ldots, n, k=$ $1,2, \ldots, l$.

As in Lemma 2.2, we define an operator $\mathcal{F}: \mathcal{C} \rightarrow \mathcal{C}$ by

$$
\begin{aligned}
(\mathcal{F} u)(t)= & I^{q} f(s, u(s))(t) \\
& +\frac{\left(\Omega_{6}-\Omega_{4}\right) t-\left(\Omega_{5}-\Omega_{3}\right)}{\Delta}\left(\omega-\sum_{i=1}^{m} \theta_{i} I^{\alpha_{i}+q} f(s, u(s))(T)\right) \\
& +\frac{\Omega_{1}-\Omega_{2} t}{\Delta}\left(\sum_{j=1}^{n} \lambda_{j} I^{\beta_{j}+q} f(s, u(s))\left(\eta_{j}\right)\right. \\
& \left.-\sum_{k=1}^{l} \mu_{k}\left(I^{\gamma_{k}+q} f(s, u(s))(T)-I^{\gamma_{k}+q} f(s, u(s))\left(\xi_{k}\right)\right)\right) .
\end{aligned}
$$

It should be noticed that problem (1.1) has solutions if and only if the operator $\mathcal{F}$ has fixed points.

In the following subsections we prove existence, as well as existence and uniqueness results, for the boundary value problem (1.1) by using a variety of fixed point theorems.

We set

$$
\begin{aligned}
\Lambda= & \frac{T^{q}}{\Gamma(q+1)}+\frac{\left(\left|\Omega_{3}\right|+\left|\Omega_{5}\right|\right)+\left(\left|\Omega_{6}\right|+\left|\Omega_{4}\right|\right) T}{|\Delta|} \sum_{i=1}^{m}\left|\theta_{i}\right| \frac{T^{\alpha_{i}+q}}{\Gamma\left(\alpha_{i}+q+1\right)} \\
& +\frac{\left|\Omega_{1}\right|+\left|\Omega_{2}\right| T}{|\Delta|}\left(\sum_{j=1}^{n}\left|\lambda_{j}\right| \frac{\eta_{j}^{\beta_{j}+q}}{\Gamma\left(\beta_{j}+q+1\right)}+\sum_{k=1}^{l}\left|\mu_{k}\right|\left(\frac{T^{\gamma_{k}+q}+\xi_{k}^{\gamma_{k}+q}}{\Gamma\left(\gamma_{k}+q+1\right)}\right)\right)
\end{aligned}
$$


and

$$
\Phi=\frac{\left(\left|\Omega_{5}\right|+\left|\Omega_{3}\right|\right)+\left(\left|\Omega_{6}\right|+\left|\Omega_{4}\right|\right) T}{|\Delta|}|\omega| .
$$

\subsection{Existence and uniqueness result via Banach's fixed point theorem}

\section{Theorem 3.1 Assume that}

$\left(\mathrm{H}_{1}\right)$ there exists a constant $L>0$ such that $|f(t, u)-f(t, v)| \leq L|u-v|$, for each $t \in[0, T]$ and $u, v \in \mathbb{R}$.

If

$$
L \Lambda<1
$$

where $\Lambda$ is defined by (3.2), then the boundary value problem (1.1) has a unique solution on $[0, T]$.

Proof We transform the problem (1.1) into a fixed point problem, $u=\mathcal{F} u$, where the operator $\mathcal{F}$ is defined as in (3.1). Observe that the fixed points of the operator $\mathcal{F}$ are solutions of problem (1.1). Applying Banach's contraction mapping principle, we shall show that $\mathcal{F}$ has a unique fixed point.

We let $\sup _{t \in[0, T]}|f(t, 0)|=M<\infty$ and choose

$$
r \geq \frac{\Lambda M+\Phi}{1-L \Lambda}
$$

where a constant $\Phi$ is defined by (3.3).

Now, we show that $\mathcal{F} B_{r} \subset B_{r}$, where $B_{r}=\{u \in \mathcal{C}:\|u\| \leq r\}$. For any $u \in B_{r}$, we have

$$
\begin{aligned}
& |(\mathcal{F} u)(t)| \\
& \leq \sup _{t \in[0, T]}\left\{I^{q}|f(s, u(s))|(t)+\frac{\left(\left|\Omega_{5}\right|+\left|\Omega_{3}\right|\right)+\left(\left|\Omega_{6}\right|+\left|\Omega_{4}\right|\right) t}{|\Delta|}|\omega|\right. \\
& +\frac{\left(\left|\Omega_{5}\right|+\left|\Omega_{3}\right|\right)+\left(\left|\Omega_{6}\right|+\left|\Omega_{4}\right|\right) t}{|\Delta|} \sum_{i=1}^{m}\left|\theta_{i}\right| I^{\alpha_{i}+q}|f(s, u(s))|(T) \\
& +\frac{\left|\Omega_{1}\right|+\left|\Omega_{2}\right| t}{|\Delta|}\left(\sum_{j=1}^{n}\left|\lambda_{j}\right| I^{\beta_{j}+q}|f(s, u(s))|\left(\eta_{j}\right)\right. \\
& \left.\left.+\sum_{k=1}^{l}\left|\mu_{k}\right|\left(I^{\gamma_{k}+q}|f(s, u(s))|(T)+I^{\gamma_{k}+q}|f(s, u(s))|\left(\xi_{k}\right)\right)\right)\right\} \\
& \leq I^{q}(|f(s, u(s))-f(s, 0)|+|f(s, 0)|)(T)+\frac{\left(\left|\Omega_{5}\right|+\left|\Omega_{3}\right|\right)+\left(\left|\Omega_{6}\right|+\left|\Omega_{4}\right|\right) T}{|\Delta|}|\omega| \\
& +\frac{\left(\left|\Omega_{5}\right|+\left|\Omega_{3}\right|\right)+\left(\left|\Omega_{6}\right|+\left|\Omega_{4}\right|\right) T}{|\Delta|} \sum_{i=1}^{m}\left|\theta_{i}\right| I^{\alpha_{i}+q}(|f(s, u(s))-f(s, 0)|+|f(s, 0)|)(T) \\
& +\frac{\left|\Omega_{1}\right|+\left|\Omega_{2}\right| T}{|\Delta|}\left(\sum_{j=1}^{n}\left|\lambda_{j}\right| I^{\beta_{j}+q}(|f(s, u(s))-f(s, 0)|+|f(s, 0)|)\left(\eta_{j}\right)\right.
\end{aligned}
$$




$$
\begin{aligned}
& +\sum_{k=1}^{l}\left|\mu_{k}\right|\left(I^{\gamma_{k}+q}(|f(s, u(s))-f(s, 0)|+|f(s, 0)|)(T)\right. \\
& \left.\left.+I^{\gamma_{k}+q}(|f(s, u(s))-f(s, 0)|+|f(s, 0)|)\left(\xi_{k}\right)\right)\right) \\
\leq & (L r+M) I^{q}(1)(T)+\frac{\left(\left|\Omega_{5}\right|+\left|\Omega_{3}\right|\right)+\left(\left|\Omega_{6}\right|+\left|\Omega_{4}\right|\right) T}{|\Delta|}|\omega| \\
& +(L r+M) \frac{\left(\left|\Omega_{5}\right|+\left|\Omega_{3}\right|\right)+\left(\left|\Omega_{6}\right|+\left|\Omega_{4}\right|\right) T}{|\Delta|} \sum_{i=1}^{m}\left|\theta_{i}\right| I^{\alpha_{i}+q}(1)(T) \\
& +(L r+M) \frac{\left|\Omega_{1}\right|+\left|\Omega_{2}\right| T}{|\Delta|}\left(\sum_{j=1}^{n}\left|\lambda_{j}\right| I^{\beta_{j}+q}(1)\left(\eta_{j}\right)\right. \\
& \left.+\sum_{k=1}^{l}\left|\mu_{k}\right|\left(I^{\gamma_{k}+q}(1)(T)+I^{\gamma_{k}+q}(1)\left(\xi_{k}\right)\right)\right) \\
\leq & (L r+M) \frac{T^{q}}{\Gamma(q+1)}+\frac{\left(\left|\Omega_{5}\right|+\left|\Omega_{3}\right|\right)+\left(\left|\Omega_{6}\right|+\left|\Omega_{4}\right|\right) T}{|\Delta|}|\omega| \\
& +(L r+M) \frac{\left(\left|\Omega_{5}\right|+\left|\Omega_{3}\right|\right)+\left(\left|\Omega_{6}\right|+\left|\Omega_{4}\right|\right) T}{|\Delta|} \sum_{i=1}^{m}\left|\theta_{i}\right| \frac{T^{\alpha_{i}+q}}{\Gamma\left(\alpha_{i}+q+1\right)} \\
& +(L r+M) \frac{\left|\Omega_{1}\right|+\left|\Omega_{2}\right| T}{|\Delta|}\left(\sum_{j=1}^{n}\left|\lambda_{j}\right| \frac{\eta_{j}^{\beta_{j}+q}}{\Gamma\left(\beta_{j}+q+1\right)}+\sum_{k=1}^{l}\left|\mu_{k}\right|\left(\frac{T^{\gamma_{k}+q}+\xi_{k}^{\gamma_{k}+q}}{\Gamma\left(\gamma_{k}+q+1\right)}\right)\right) \\
= & (L r+M) \Lambda+\Phi \leq r,
\end{aligned}
$$

which implies that $\mathcal{F} B_{r} \subset B_{r}$.

Next, we let $u, v \in \mathcal{C}$. Then for $t \in[0, T]$, we have

$$
\begin{aligned}
|(\mathcal{F} u)(t)-(\mathcal{F} v)(t)| & \leq I^{q}|f(s, u(s))-f(s, v(s))|(t) \\
& +\frac{\left(\left|\Omega_{5}\right|+\left|\Omega_{3}\right|\right)+\left(\left|\Omega_{6}\right|+\left|\Omega_{4}\right|\right) T}{|\Delta|} \sum_{i=1}^{m}\left|\theta_{i}\right| I^{\alpha_{i}+q}|f(s, u(s))-f(s, v(s))|(T) \\
& +\frac{\left|\Omega_{1}\right|+\left|\Omega_{2}\right| T}{|\Delta|}\left(\sum_{j=1}^{n}\left|\lambda_{j}\right| I^{\beta_{j}+q}|f(s, u(s))-f(s, v(s))|\left(\eta_{j}\right)\right. \\
& \left.+\sum_{k=1}^{l}\left|\mu_{k}\right|\left(I^{\gamma_{k}+q}|f(s, u(s))-f(s, v(s))|(T)+I^{\gamma_{k}+q}|f(s, u(s))-f(s, v(s))|\left(\xi_{k}\right)\right)\right) \\
\leq & L\|u-v\| \frac{T^{q}}{\Gamma(q+1)} \\
& +L\|u-v\| \frac{\left(\left|\Omega_{5}\right|+\left|\Omega_{3}\right|\right)+\left(\left|\Omega_{6}\right|+\left|\Omega_{4}\right|\right) T}{|\Delta|} \sum_{i=1}^{m}\left|\theta_{i}\right| \frac{T^{\alpha_{i}+q}}{\Gamma\left(\alpha_{i}+q+1\right)} \\
& +L\|u-v\| \frac{\left|\Omega_{1}\right|+\left|\Omega_{2}\right| T}{|\Delta|}\left(\sum_{j=1}^{n}\left|\lambda_{j}\right| \frac{\eta_{j}^{\beta_{j}+q}}{\Gamma\left(\beta_{j}+q+1\right)}+\sum_{k=1}^{l}\left|\mu_{k}\right|\left(\frac{T^{\gamma_{k}+q}+\xi_{k}^{\gamma_{k}+q}}{\Gamma\left(\gamma_{k}+q+1\right)}\right)\right) \\
= & L \Lambda\|u-v\|, \quad
\end{aligned}
$$


which implies that $\|\mathcal{F} u-\mathcal{F} v\| \leq L \Lambda\|u-v\|$. As $L \Lambda<1, \mathcal{F}$ is a contraction. Therefore, we deduce, by Banach's contraction mapping principle, that $\mathcal{F}$ has a fixed point which is the unique solution of problem (1.1). The proof is completed.

\subsection{Existence and uniqueness result via Banach's fixed point theorem and Hölder inequality}

Theorem 3.2 Suppose that $f:[0, T] \times \mathbb{R} \rightarrow \mathbb{R}$ is a continuous function satisfying the following assumption:

$\left(\mathrm{H}_{2}\right)|f(t, u)-f(t, v)| \leq \delta(t)|u-v|$, for $t \in[0, T], u, v \in \mathbb{R}$ and $\delta \in L^{\frac{1}{\sigma}}\left([0, T], \mathbb{R}^{+}\right), \sigma \in(0,1)$.

Denote $\|\delta\|=\left(\int_{0}^{T}|\delta(s)|^{\frac{1}{\sigma}} d s\right)^{\sigma}$. If

$$
\begin{aligned}
& \|\delta\|\left\{\frac{T^{q-\sigma}}{\Gamma(q)}\left(\frac{1-\sigma}{q-\sigma}\right)^{1-\sigma}+\frac{\left(\left|\Omega_{6}\right|+\left|\Omega_{4}\right|\right) T+\left(\left|\Omega_{5}\right|+\left|\Omega_{3}\right|\right)}{|\Delta|}\right. \\
& \quad \times \sum_{i=1}^{m} \frac{\left|\theta_{i}\right| T^{\alpha_{i}+q-\sigma}}{\Gamma\left(\alpha_{i}+q\right)}\left(\frac{1-\sigma}{\alpha_{i}+q-\sigma}\right)^{1-\sigma}+\frac{\left|\Omega_{1}\right|+\left|\Omega_{2}\right| T}{|\Delta|}\left(\sum_{j=1}^{n} \frac{\left|\lambda_{j}\right| \eta^{\beta_{j}+q-\sigma}}{\Gamma\left(\beta_{j}+q\right)}\left(\frac{1-\sigma}{\beta_{j}+q-\sigma}\right)^{1-\sigma}\right. \\
& \left.\left.\quad+\sum_{k=1}^{l} \frac{\left|\mu_{k}\right|}{\Gamma\left(\beta_{j}+q\right)}\left(T^{\gamma_{k}+q-\sigma}+\xi_{k}^{\gamma_{k}+q-\sigma}\right)\left(\frac{1-\sigma}{\gamma_{k}+q-\sigma}\right)^{1-\sigma}\right)\right\}<1,
\end{aligned}
$$

then the boundary value problem (1.1) has a unique solution.

Proof For $u, v \in C([0, T], \mathbb{R})$ and for each $t \in[0, T]$, by Hölder's inequality, we have

$$
\begin{aligned}
|(\mathcal{F} u)(t)-(\mathcal{F} v)(t)| & \leq I^{q}|f(s, u(s))-f(s, v(s))|(t) \\
& +\frac{\left(\left|\Omega_{5}\right|+\left|\Omega_{3}\right|\right)+\left(\left|\Omega_{6}\right|+\left|\Omega_{4}\right|\right) T}{|\Delta|} \sum_{i=1}^{m}\left|\theta_{i}\right| I^{\alpha_{i}+q}|f(s, u(s))-f(s, v(s))|(T) \\
& +\frac{\left|\Omega_{1}\right|+\left|\Omega_{2}\right| T}{|\Delta|}\left(\sum_{j=1}^{n}\left|\lambda_{j}\right| I^{\beta_{j}+q}|f(s, u(s))-f(s, v(s))|\left(\eta_{j}\right)\right. \\
& \left.+\sum_{k=1}^{l}\left|\mu_{k}\right|\left(I^{\gamma_{k}+q}|f(s, u(s))-f(s, v(s))|(T)+I^{\gamma_{k}+q}|f(s, u(s))-f(s, v(s))|\left(\xi_{k}\right)\right)\right) \\
\leq & \frac{1}{\Gamma(q)} \int_{0}^{t}(t-s)^{q-1} \delta(s)|u(s)-v(s)| d s \\
& +\frac{\left(\left|\Omega_{5}\right|+\left|\Omega_{3}\right|\right)+\left(\left|\Omega_{6}\right|+\left|\Omega_{4}\right|\right) T}{|\Delta|} \sum_{i=1}^{m} \frac{\left|\theta_{i}\right|}{\Gamma\left(\alpha_{i}+q\right)} \int_{0}^{T}(T-s)^{\alpha_{i}+q-1} \delta(s)|u(s)-v(s)| d s \\
& +\frac{\left|\Omega_{1}\right|+\left|\Omega_{2}\right| T}{|\Delta|}\left(\sum_{j=1}^{n} \frac{\left|\lambda_{j}\right|}{\Gamma\left(\eta_{j}+q\right)} \int_{0}^{\eta_{j}}\left(\eta_{j}-s\right)^{\eta_{j}+q-1} \delta(s)|u(s)-v(s)| d s\right. \\
& +\sum_{k=1}^{l} \frac{\left|\mu_{k}\right|}{\Gamma\left(\gamma_{k}+q\right)}\left(\int_{0}^{T}(T-s)^{\gamma_{k}+q-1} \delta(s)|u(s)-v(s)| d s\right. \\
& \left.\left.+\int_{0}^{\xi_{k}}\left(\xi_{k}-s\right)^{\gamma_{k}+q-1} \delta(s)|u(s)-v(s)| d s\right)\right)
\end{aligned}
$$




$$
\begin{aligned}
\leq & \frac{1}{\Gamma(q)}\left(\int_{0}^{t}\left((t-s)^{q-1}\right)^{\frac{1}{1-\sigma}} d s\right)^{1-\sigma}\left(\int_{0}^{t}(\delta(s))^{\frac{1}{\sigma}} d s\right)^{\sigma}\|u-v\| \\
& +\frac{\left(\left|\Omega_{5}\right|+\left|\Omega_{3}\right|\right)+\left(\left|\Omega_{6}\right|+\left|\Omega_{4}\right|\right) T}{|\Delta|} \\
& \times \sum_{i=1}^{m} \frac{\left|\theta_{i}\right|}{\Gamma\left(\alpha_{i}+q\right)}\left(\int_{0}^{T}\left((T-s)^{\alpha_{i}+q-1}\right)^{\frac{1}{1-\sigma}} d s\right)^{1-\sigma}\left(\int_{0}^{T}(\delta(s))^{\frac{1}{\sigma}} d s\right)^{\sigma}\|u-v\| \\
& +\frac{\left|\Omega_{1}\right|+\left|\Omega_{2}\right| T}{|\Delta|}\left(\sum_{j=1}^{n} \frac{\left|\lambda_{j}\right|}{\Gamma\left(\eta_{j}+q\right)}\left(\int_{0}^{\eta_{j}}\left(\left(\eta_{j}-s\right)^{\beta_{j}+q-1}\right)^{\frac{1}{1-\sigma}} d s\right)^{1-\sigma}\left(\int_{0}^{\eta_{j}}(\delta(s))^{\frac{1}{\sigma}} d s\right)^{\sigma}\right. \\
& +\sum_{k=1}^{l} \frac{\left|\mu_{k}\right|}{\Gamma\left(\gamma_{k}+q\right)}\left(\left(\int_{0}^{T}\left((T-s)^{\gamma_{k}+q-1}\right)^{\frac{1}{1-\sigma}} d s\right)^{1-\sigma}\left(\int_{0}^{T}(\delta(s))^{\frac{1}{\sigma}} d s\right)^{\sigma}\right. \\
& \left.\left.+\left(\int_{0}^{\xi_{k}}\left(\left(\xi_{k}-s\right)^{\gamma_{k}+q-1}\right)^{\frac{1}{1-\sigma}} d s\right)^{1-\sigma}\left(\int_{0}^{\xi_{k}}(\delta(s))^{\frac{1}{\sigma}} d s\right)^{\sigma}\right)\right)\|u-v\| \\
\leq & \|\delta\|\left[\frac{T^{q-\sigma}}{\Gamma(q)}\left(\frac{1-\sigma}{q-\sigma}\right)^{1-\sigma}+\frac{\left(\left|\Omega_{5}\right|+\left|\Omega_{3}\right|\right)+\left(\left|\Omega_{6}\right|+\left|\Omega_{4}\right|\right) T}{|\Delta|} \sum_{i=1}^{m} \frac{\left|\theta_{i}\right| T^{\alpha_{i}+q-\sigma}}{\Gamma\left(\alpha_{i}+q\right)}\right. \\
& \times\left(\frac{1-\sigma}{\alpha_{i}+q-\sigma}\right)^{1-\sigma}+\frac{\left|\Omega_{1}\right|+\left|\Omega_{2}\right| T}{|\Delta|}\left(\sum_{j=1}^{n} \frac{\left|\lambda_{j}\right| \eta_{j}^{\beta_{j}+q-\sigma}}{\Gamma\left(\eta_{j}+q\right)}\left(\frac{1-\sigma}{\beta_{j}+q-\sigma}\right)^{1-\sigma}\right. \\
& \left.\left.+\sum_{k=1}^{l} \frac{\left|\mu_{k}\right|}{\Gamma\left(\gamma_{k}+q\right)}\left(T^{\gamma_{k}+q-\sigma}+\xi_{k}^{\gamma_{k}+q-\sigma}\right)\left(\frac{1-\sigma}{\gamma_{k}+q-\sigma}\right)^{1-\sigma}\right)\right]\|u-v\| .
\end{aligned}
$$

It follows that $\mathcal{F}$ is contraction mapping. Hence Banach's fixed point theorem implies that $\mathcal{F}$ has a unique fixed point, which is the unique solution of the problem (1.1). The proof is completed.

\subsection{Existence and uniqueness result via nonlinear contractions}

Definition 3.1 Let $E$ be a Banach space and let $F: E \rightarrow E$ be a mapping. $F$ is said to be a nonlinear contraction if there exists a continuous nondecreasing function $\Psi: \mathbb{R}^{+} \rightarrow \mathbb{R}^{+}$ such that $\Psi(0)=0$ and $\Psi(\varepsilon)<\varepsilon$ for all $\varepsilon>0$ with the property

$$
\|F u-F v\| \leq \Psi(\|u-v\|), \quad \forall u, v \in E .
$$

Lemma 3.1 (Boyd and Wong [22]) Let E be a Banach space and let $F: E \rightarrow E$ be a nonlinear contraction. Then $F$ has a unique fixed point in $E$.

Theorem 3.3 Let $f:[0, T] \times \mathbb{R} \rightarrow \mathbb{R}$ be a continuous function satisfying the assumption

$\left(\mathrm{H}_{3}\right)|f(t, u)-f(t, v)| \leq h(t) \frac{|u-v|}{H^{*}+|u-v|}, t \in[0, T], u, v \geq 0$, where $h:[0, T] \rightarrow \mathbb{R}^{+}$is continuous and a constant $H^{*}$ defined by

$$
\begin{aligned}
H^{*}= & I^{q} h(T)+\frac{\left(\left|\Omega_{5}\right|+\left|\Omega_{3}\right|\right)+\left(\left|\Omega_{6}\right|+\left|\Omega_{4}\right|\right) T}{|\Delta|} \sum_{i=1}^{m}\left|\theta_{i}\right| I^{\alpha_{i}+q} h(T)+\frac{\left|\Omega_{1}\right|+\left|\Omega_{2}\right| T}{|\Delta|} \\
& \times\left(\sum_{j=1}^{n}\left|\lambda_{j}\right| I^{\beta_{j}+q} h\left(\eta_{j}\right)+\sum_{k=1}^{l}\left|\mu_{k}\right|\left(I^{\gamma_{k}+q} h(T)+I^{\gamma_{k}+q} h\left(\xi_{k}\right)\right)\right) .
\end{aligned}
$$

Then the boundary value problem (1.1) has a unique solution. 
Proof We define the operator $\mathcal{F}: \mathcal{C} \rightarrow \mathcal{C}$ as (3.1) and a continuous nondecreasing function $\Psi: \mathbb{R}^{+} \rightarrow \mathbb{R}^{+}$by

$$
\Psi(\varepsilon)=\frac{H^{*} \varepsilon}{H^{*}+\varepsilon}, \quad \forall \varepsilon \geq 0 .
$$

Note that the function $\Psi$ satisfies $\Psi(0)=0$ and $\Psi(\varepsilon)<\varepsilon$ for all $\varepsilon>0$.

For any $u, v \in \mathcal{C}$ and for each $t \in[0, T]$, we have

$$
\begin{aligned}
|(\mathcal{F} u)(t)-(\mathcal{F} v)(t)| & \\
\leq & I^{q}|f(s, u(s))-f(s, v(s))|(t) \\
& +\frac{\left(\left|\Omega_{5}\right|+\left|\Omega_{3}\right|\right)+\left(\left|\Omega_{6}\right|+\left|\Omega_{4}\right|\right) T}{|\Delta|} \sum_{i=1}^{m}\left|\theta_{i}\right| I^{\alpha_{i}+q}|f(s, u(s))-f(s, v(s))|(T) \\
& +\frac{\left|\Omega_{1}\right|+\left|\Omega_{2}\right| T}{|\Delta|}\left(\sum_{j=1}^{n}\left|\lambda_{j}\right| I^{\beta_{j}+q}|f(s, u(s))-f(s, v(s))|\left(\eta_{j}\right)\right. \\
& \left.+\sum_{k=1}^{l}\left|\mu_{k}\right|\left(I^{\gamma_{k}+q}|f(s, u(s))-f(s, v(s))|(T)+I^{\gamma_{k}+q}|f(s, u(s))-f(s, v(s))|\left(\xi_{k}\right)\right)\right) \\
\leq & I^{q}\left(h(s) \frac{|u-v|}{H^{*}+|u-v|}\right)(T) \\
& +\frac{\left(\left|\Omega_{5}\right|+\left|\Omega_{3}\right|\right)+\left(\left|\Omega_{6}\right|+\left|\Omega_{4}\right|\right) T}{|\Delta|} \sum_{i=1}^{m}\left|\theta_{i}\right| I^{\alpha_{i}+q}\left(h(s) \frac{|u-v|}{H^{*}+|u-v|}\right)(T) \\
& +\frac{\left|\Omega_{1}\right|+\left|\Omega_{2}\right| T}{|\Delta|}\left(\sum_{j=1}^{n}\left|\lambda_{j}\right| I^{\beta_{j}+q}\left(h(s) \frac{|u-v|}{H^{*}+|u-v|}\right)\left(\eta_{j}\right)\right. \\
& \left.+\sum_{k=1}^{l}\left|\mu_{k}\right|\left(I^{\gamma_{k}+q}\left(h(s) \frac{|u-v|}{H^{*}+|u-v|}\right)(T)+I^{\gamma_{k}+q}\left(h(s) \frac{|u-v|}{H^{*}+|u-v|}\right)\left(\xi_{k}\right)\right)\right) \\
\leq & \frac{\Psi\|u-v\|}{H^{*}}\left(I^{q} h(T)+\frac{\left(\left|\Omega_{5}\right|+\left|\Omega_{3}\right|\right)+\left(\left|\Omega_{6}\right|+\left|\Omega_{4}\right|\right) T}{|\Delta|} \sum_{i=1}^{m}\left|\theta_{i}\right| I^{\alpha_{i}+q} h(T)\right. \\
& \left.+\frac{\left|\Omega_{1}\right|+\left|\Omega_{2}\right| T}{|\Delta|}\left(\sum_{j=1}^{n}\left|\lambda_{j}\right| I^{\beta_{j}+q} h\left(\eta_{j}\right)+\sum_{k=1}^{l}\left|\mu_{k}\right|\left(I^{\gamma_{k}+q} h(T)+I^{\gamma_{k}+q} h\left(\xi_{k}\right)\right)\right)\right) \\
&
\end{aligned}
$$

This implies that $\|\mathcal{F} u-\mathcal{F} v\| \leq \Psi(\|u-v\|)$. Therefore $\mathcal{F}$ is a nonlinear contraction. Hence, by Lemma 3.1 the operator $\mathcal{F}$ has a unique fixed point which is the unique solution of the boundary value problem (1.1). This completes the proof.

\subsection{Existence result via Krasnoselskii's fixed point theorem}

Lemma 3.2 (Krasnoselskii's fixed point theorem [23]) Let $M$ be a closed, bounded, convex, and nonempty subset of a Banach space X. Let A, B be the operators such that (a) $A x+B y \in$ $M$ whenever $x, y \in M$; (b) $A$ is compact and continuous; (c) $B$ is a contraction mapping. Then there exists $z \in M$ such that $z=A z+B z$. 
Theorem 3.4 Let $f:[0, T] \times \mathbb{R} \rightarrow \mathbb{R}$ be a continuous function satisfying $\left(\mathrm{H}_{1}\right)$. In addition we assume that

$\left(\mathrm{H}_{4}\right)|f(t, u)| \leq \phi(t), \forall(t, u) \in[0, T] \times \mathbb{R}$, and $\phi \in C\left([0, T], \mathbb{R}^{+}\right)$.

Then the boundary value problem (1.1) has at least one solution on $[0, T]$ provided

$$
\begin{aligned}
& \frac{\left(\left|\Omega_{3}\right|+\left|\Omega_{5}\right|\right)+\left(\left|\Omega_{6}\right|+\left|\Omega_{4}\right|\right) T}{|\Delta|} \sum_{i=1}^{m}\left|\theta_{i}\right| \frac{T^{\alpha_{i}+q}}{\Gamma\left(\alpha_{i}+q+1\right)} \\
& +\frac{\left|\Omega_{1}\right|+\left|\Omega_{2}\right| T}{|\Delta|}\left(\sum_{j=1}^{n}\left|\lambda_{j}\right| \frac{\eta_{j}^{\beta_{j}+q}}{\Gamma\left(\beta_{j}+q+1\right)}+\sum_{k=1}^{l}\left|\mu_{k}\right|\left(\frac{T^{\gamma_{k}+q}+\xi_{k}^{\gamma_{k}+q}}{\Gamma\left(\gamma_{k}+q+1\right)}\right)\right)<1 .
\end{aligned}
$$

Proof Setting $\sup _{t \in[0, T]}|\varphi(t)|=\|\varphi\|$ and choosing

$$
\rho \geq\|\varphi\| \Lambda+\Phi
$$

(where $\Lambda$ and $\Phi$ are defined by (3.2) and (3.3), respectively), we consider $B_{\rho}=\{u \in$ $\mathcal{C}([0, T], \mathbb{R}):\|u\| \leq \rho\}$. We define the operators $\mathcal{F}_{1}$ and $\mathcal{F}_{2}$ on $B_{\rho}$ by

$$
\begin{aligned}
\mathcal{F}_{1} u(t)= & I^{q} f(s, u(s))(t), \quad t \in[0, T], \\
\mathcal{F}_{2} u(t)= & \frac{\left(\Omega_{6}-\Omega_{4}\right) t-\left(\Omega_{5}-\Omega_{3}\right)}{\Delta}\left(\omega_{1}-\sum_{i=1}^{m} \theta_{i} I^{\alpha_{i}+q} f(s, u(s))(T)\right) \\
& +\frac{\Omega_{1}-\Omega_{2} t}{\Delta}\left(\sum_{j=1}^{n} \lambda_{j} I^{\beta_{j}+q} f(s, u(s))\left(\eta_{j}\right)\right. \\
& \left.-\sum_{k=1}^{l} \mu_{k}\left(I^{\gamma_{k}+q} f(s, u(s))(T)-I^{\gamma_{k}+q} f(s, u(s))\left(\xi_{k}\right)\right)\right), \quad t \in[0, T] .
\end{aligned}
$$

For any $x, y \in B_{\rho}$, we have

$$
\begin{aligned}
& \left|\mathcal{F}_{1} u(t)+\mathcal{F}_{2} v(t)\right| \\
& \leq \sup _{t \in[0, T]}\left\{I^{q}|f(s, u(s))|(t)+\frac{\left(\left|\Omega_{5}\right|+\left|\Omega_{3}\right|\right)+\left(\left|\Omega_{6}\right|+\left|\Omega_{4}\right|\right) t}{|\Delta|}|\omega|\right. \\
& \quad+\frac{\left(\left|\Omega_{5}\right|+\left|\Omega_{3}\right|\right)+\left(\left|\Omega_{6}\right|+\left|\Omega_{4}\right|\right) t}{|\Delta|} \sum_{i=1}^{m}\left|\theta_{i}\right| I^{\alpha_{i}+q}|f(s, v(s))|(T) \\
& \quad+\frac{\left|\Omega_{1}\right|+\left|\Omega_{2}\right| t}{|\Delta|}\left(\sum_{j=1}^{n}\left|\lambda_{j}\right| I^{\beta_{j}+q}|f(s, v(s))|\left(\eta_{j}\right)\right. \\
& \left.\left.\quad+\sum_{k=1}^{l}\left|\mu_{k}\right|\left(I^{\gamma_{k}+q}|f(s, v(s))|(T)+I^{\gamma_{k}+q}|f(s, v(s))|\left(\xi_{k}\right)\right)\right)\right\} \\
& \leq\|\varphi\|\left[\frac{T^{q}}{\Gamma(q+1)}+\frac{\left(\left|\Omega_{5}\right|+\left|\Omega_{3}\right|\right)+\left(\left|\Omega_{6}\right|+\left|\Omega_{4}\right|\right) T}{|\Delta|} \sum_{i=1}^{m}\left|\theta_{i}\right| \frac{T^{\alpha_{i}+q}}{\Gamma\left(\alpha_{i}+q+1\right)}\right. \\
& \left.\quad+\frac{\left|\Omega_{1}\right|+\left|\Omega_{2}\right| T}{|\Delta|}\left(\sum_{j=1}^{n}\left|\lambda_{j}\right| \frac{\eta_{j}^{\beta_{j}+q}}{\Gamma\left(\beta_{j}+q+1\right)}+\sum_{k=1}^{l}\left|\mu_{k}\right|\left(\frac{T^{\gamma_{k}+q}+\xi_{k}^{\gamma_{k}+q}}{\Gamma\left(\gamma_{k}+q+1\right)}\right)\right)\right]
\end{aligned}
$$




$$
\begin{aligned}
& \quad+\frac{\left(\left|\Omega_{5}\right|+\left|\Omega_{3}\right|\right)+\left(\left|\Omega_{6}\right|+\left|\Omega_{4}\right|\right) T}{|\Delta|}|\omega| \\
& =\|\varphi\| \Lambda+\Phi \leq \rho .
\end{aligned}
$$

This shows that $\mathcal{F}_{1} u+\mathcal{F}_{2} v \in B_{\rho}$. It is easy to see using (3.6) that $\mathcal{F}_{2}$ is a contraction mapping.

Continuity of $f$ implies that the operator $\mathcal{F}_{1}$ is continuous. Also, $\mathcal{F}_{1}$ is uniformly bounded on $B_{\rho}$ as

$$
\left\|\mathcal{F}_{1} u\right\| \leq \frac{T^{q}}{\Gamma(q+1)}\|\phi\|
$$

Now we prove the compactness of the operator $\mathcal{F}_{1}$.

We define $\sup _{(t, u) \in[0, T] \times B_{\rho}}|f(t, u)|=\bar{f}<\infty$, and consequently we have

$$
\begin{aligned}
\left|\left(\mathcal{F}_{1} u\right)\left(t_{2}\right)-\left(\mathcal{F}_{1} u\right)\left(t_{1}\right)\right|= & \frac{1}{\Gamma(q)} \mid \int_{0}^{t_{1}}\left[\left(t_{2}-s\right)^{q-1}-\left(t_{1}-s\right)^{q-1}\right] f(s, u(s)) d s \\
& +\int_{t_{1}}^{t_{2}}\left(t_{2}-s\right)^{q-1} f(s, u(s)) d s \mid \\
\leq & \frac{\bar{f}}{\Gamma(q+1)}\left|t_{1}^{q}-t_{2}^{q}\right|,
\end{aligned}
$$

which is independent of $u$ and tends to zero as $t_{2}-t_{2} \rightarrow 0$. Thus, $\mathcal{F}_{1}$ is equicontinuous. So $\mathcal{F}_{1}$ is relatively compact on $B_{\rho}$. Hence, by the Arzelá-Ascoli theorem, $\mathcal{F}_{1}$ is compact on $B_{\rho}$. Thus all the assumptions of Lemma 3.2 are satisfied. So the conclusion of Lemma 3.2 implies that the boundary value problem (1.1) has at least one solution on $[0, T]$.

\subsection{Existence result via Leray-Schauder's nonlinear alternative}

Theorem 3.5 (Nonlinear alternative for single valued maps [24]) Let E be a Banach space, $C$ a closed, convex subset of $E, U$ an open subset of $C$, and $0 \in U$. Suppose that $F: \bar{U} \rightarrow C$ is a continuous, compact (that is, $F(\bar{U})$ is a relatively compact subset of $C$ ) map. Then either

(i) F has a fixed point in $\bar{U}$, or

(ii) there is a $u \in \partial U$ (the boundary of $U$ in $C$ ) and $\lambda \in(0,1)$ with $u=\lambda F(u$ ).

\section{Theorem 3.6 Assume that}

$\left(\mathrm{H}_{5}\right)$ there exist a continuous nondecreasing function $\psi:[0, \infty) \rightarrow(0, \infty)$ and a function $p \in C\left([0, T], \mathbb{R}^{+}\right)$such that

$$
|f(t, u)| \leq p(t) \psi(|u|) \quad \text { for each }(t, u) \in[0, T] \times \mathbb{R} ;
$$

$\left(\mathrm{H}_{6}\right)$ there exists a constant $M>0$ such that

$$
\frac{M}{\psi(M)\|p\| \Lambda+\Phi}>1
$$

where $\Lambda$ and $\Phi$ are defined by (3.2) and (3.3), respectively.

Then the boundary value problem (1.1) has at least one solution on $[0, T]$. 
Proof Let the operator $\mathcal{F}$ be defined by (3.1). Firstly, we shall show that $\mathcal{F}$ maps bounded sets (balls) into bounded sets in $C([0, T], \mathbb{R})$. For a number $r>0$, let $B_{r}=\{u \in C([0, T], \mathbb{R})$ : $\|u\| \leq r\}$ be a bounded ball in $C([0, T], \mathbb{R})$. Then for $t \in[0, T]$ we have

$$
\begin{aligned}
& |(\mathcal{F} u)(t)| \\
& \leq \sup _{t \in[0, T]}\left\{I^{q}|f(s, u(s))|(t)+\frac{\left(\left|\Omega_{5}\right|+\left|\Omega_{3}\right|\right)+\left(\left|\Omega_{6}\right|+\left|\Omega_{4}\right|\right) t}{|\Delta|}|\omega|\right. \\
& +\frac{\left(\left|\Omega_{5}\right|+\left|\Omega_{3}\right|\right)+\left(\left|\Omega_{6}\right|+\left|\Omega_{4}\right|\right) T}{|\Delta|} \sum_{i=1}^{m}\left|\theta_{i}\right| I^{\alpha_{i}+q}|f(s, u(s))|(T) \\
& +\frac{\left|\Omega_{1}\right|+\left|\Omega_{2}\right| T}{|\Delta|}\left(\sum_{j=1}^{n}\left|\lambda_{j}\right| I^{\beta_{j}+q}|f(s, u(s))|\left(\eta_{j}\right)\right. \\
& \left.\left.+\sum_{k=1}^{l}\left|\mu_{k}\right|\left(I^{\gamma_{k}+q}|f(s, u(s))|(T)+I^{\gamma_{k}+q}|f(s, u(s))|\left(\xi_{k}\right)\right)\right)\right\} \\
& \leq \psi(\|u\|) I^{q} p(s)(T)+\frac{\left(\left|\Omega_{5}\right|+\left|\Omega_{3}\right|\right)+\left(\left|\Omega_{6}\right|+\left|\Omega_{4}\right|\right) T}{|\Delta|}|\omega| \\
& +\psi(\|u\|) \frac{\left(\left|\Omega_{5}\right|+\left|\Omega_{3}\right|\right)+\left(\left|\Omega_{6}\right|+\left|\Omega_{4}\right|\right) T}{|\Delta|} \sum_{i=1}^{m}\left|\theta_{i}\right| I^{\alpha_{i}+q} p(s)(T) \\
& +\psi(\|u\|) \frac{\left|\Omega_{1}\right|+\left|\Omega_{2}\right| T}{|\Delta|}\left(\sum_{j=1}^{n}\left|\lambda_{j}\right| I^{\beta_{j}+q} p(s)\left(\eta_{j}\right)\right. \\
& \left.+\sum_{k=1}^{l}\left|\mu_{k}\right|\left(I^{\gamma_{k}+q} p(s)(T)+I^{\gamma_{k}+q} p(s)\left(\xi_{k}\right)\right)\right) \\
& \leq \psi(\|u\|)\|p\| \frac{T^{q}}{\Gamma(q+1)}+\frac{\left(\left|\Omega_{5}\right|+\left|\Omega_{3}\right|\right)+\left(\left|\Omega_{6}\right|+\left|\Omega_{4}\right|\right) T}{|\Delta|}|\omega| \\
& +\psi(\|u\|)\|p\| \frac{\left(\left|\Omega_{5}\right|+\left|\Omega_{3}\right|\right)+\left(\left|\Omega_{6}\right|+\left|\Omega_{4}\right|\right) T}{|\Delta|} \sum_{i=1}^{m}\left|\theta_{i}\right| \frac{T^{\alpha_{i}+q}}{\Gamma\left(\alpha_{i}+q+1\right)} \\
& +\psi(\|u\|)\|p\| \frac{\left|\Omega_{1}\right|+\left|\Omega_{2}\right| T}{|\Delta|} \\
& \times\left(\sum_{j=1}^{n}\left|\lambda_{j}\right| \frac{\eta_{j}^{\beta_{j}+q}}{\Gamma\left(\beta_{j}+q+1\right)}+\sum_{k=1}^{l}\left|\mu_{k}\right|\left(\frac{T^{\gamma_{k}+q}+\xi_{k}^{\gamma_{k}+q}}{\Gamma\left(\gamma_{k}+q+1\right)}\right)\right) \\
& \leq \psi(\|u\|)\|p\|\left[\frac{T^{q}}{\Gamma(q+1)}+\frac{\left(\left|\Omega_{5}\right|+\left|\Omega_{3}\right|\right)+\left(\left|\Omega_{6}\right|+\left|\Omega_{4}\right|\right) T}{|\Delta|} \sum_{i=1}^{m}\left|\theta_{i}\right| \frac{T^{\alpha_{i}+q}}{\Gamma\left(\alpha_{i}+q+1\right)}\right. \\
& \left.+\frac{\left|\Omega_{1}\right|+\left|\Omega_{2}\right| T}{|\Delta|}\left(\sum_{j=1}^{n}\left|\lambda_{j}\right| \frac{\eta_{j}^{\beta_{j}+q}}{\Gamma\left(\beta_{j}+q+1\right)}+\sum_{k=1}^{l}\left|\mu_{k}\right|\left(\frac{T^{\gamma_{k}+q}+\xi_{k}^{\gamma_{k}+q}}{\Gamma\left(\gamma_{k}+q+1\right)}\right)\right)\right] \\
& +\frac{\left(\left|\Omega_{5}\right|+\left|\Omega_{3}\right|\right)+\left(\left|\Omega_{6}\right|+\left|\Omega_{4}\right|\right) T}{|\Delta|}|\omega|,
\end{aligned}
$$

and consequently

$$
\|\mathcal{F} u\| \leq \psi(r)\|p\| \Lambda+\Phi
$$


Next we will show that $\mathcal{F}$ maps bounded sets into equicontinuous sets of $C([0, T], \mathbb{R})$. Let $\tau_{1}, \tau_{2} \in[0, T]$ with $\tau_{1}<\tau_{2}$ and $u \in B_{r}$. Then we have

$$
\begin{aligned}
\left|(\mathcal{F} u)\left(\tau_{2}\right)-(\mathcal{F} u)\left(\tau_{1}\right)\right| & \\
\leq & \frac{1}{\Gamma(q)}\left|\int_{0}^{\tau_{1}}\left[\left(\tau_{2}-s\right)^{q-1}-\left(\tau_{1}-s\right)^{q-1}\right] f(s, u(s)) d s+\int_{\tau_{1}}^{\tau_{2}}\left(\tau_{2}-s\right)^{q-1} f(s, u(s)) d s\right| \\
& +\frac{\left(\left|\Omega_{6}\right|+\left|\Omega_{4}\right|\right)\left|\omega_{1}\right|}{|\Delta|}\left(\tau_{2}-\tau_{1}\right)+\frac{\left|\Omega_{6}\right|+\left|\Omega_{4}\right|}{|\Delta|} \sum_{i=1}^{m}\left|\theta_{i}\right| I^{\alpha_{i}+q}|f(s, u(s))|(T)\left(\tau_{2}-\tau_{1}\right) \\
& +\frac{\left|\Omega_{2}\right|}{|\Delta|}\left(\tau_{2}-\tau_{1}\right)\left(\sum_{j=1}^{n}\left|\lambda_{j}\right| I^{\beta_{j}+q}|f(s, u(s))|\left(\eta_{j}\right)\right. \\
& \left.+\sum_{k=1}^{l}\left|\mu_{k}\right|\left(I^{\gamma_{k}+q}|f(s, u(s))|(T)+I^{\gamma_{k}+q}|f(s, u(s))|\left(\xi_{k}\right)\right)\right) \\
\leq & \frac{\psi(r)}{\Gamma(q)}\left|\int_{0}^{\tau_{1}}\left[\left(\tau_{2}-s\right)^{q-1}-\left(\tau_{1}-s\right)^{q-1}\right] p(s) d s+\int_{\tau_{1}}^{\tau_{2}}\left(\tau_{2}-s\right)^{q-1} p(s) d s\right| \\
& +\frac{\left(\left|\Omega_{6}\right|+\left|\Omega_{4}\right|\right)\left|\omega_{1}\right|}{|\Delta|}\left(\tau_{2}-\tau_{1}\right)+\frac{\left|\Omega_{6}\right|+\left|\Omega_{4}\right|}{|\Delta|} \psi(r) \sum_{i=1}^{m}\left|\theta_{i}\right| I^{\alpha_{i}+q} p(s)(T)\left(\tau_{2}-\tau_{1}\right) \\
& +\frac{\left|\Omega_{2}\right|}{|\Delta|} \psi(r)\left(\tau_{2}-\tau_{1}\right)\left(\sum_{j=1}^{n}\left|\lambda_{j}\right| I^{\beta_{j}+q} p(s)\left(\eta_{j}\right)\right. \\
& \left.+\sum_{k=1}^{l}\left|\mu_{k}\right|\left(I^{\gamma_{k}+q} p(s)(T)+I^{\gamma_{k}+q} p(s)\left(\xi_{k}\right)\right)\right) .
\end{aligned}
$$

As $\tau_{2}-\tau_{1} \rightarrow 0$, the right-hand side of the above inequality tends to zero independently of $u \in B_{r}$. Therefore by the Arzelá-Ascoli theorem the operator $\mathcal{F}: C([0, T], \mathbb{R}) \rightarrow$ $C([0, T], \mathbb{R})$ is completely continuous.

Let $u$ be a solution. Then, for $t \in[0, T]$, and following similar computations to those in the first step, we have

$$
|u(t)| \leq \psi(\|u\|)\|p\| \Lambda+\Phi,
$$

which leads to

$$
\frac{\|u\|}{\psi(\|u\|)\|p\| \Lambda+\Phi} \leq 1
$$

In view of $\left(\mathrm{H}_{6}\right)$, there exists $M$ such that $\|u\| \neq M$. Let us set

$$
U=\{u \in C([0, T], \mathbb{R}):\|u\|<M\} .
$$

We see that the operator $\mathcal{F}: \bar{U} \rightarrow C([0, T], \mathbb{R})$ is continuous and completely continuous. From the choice of $U$, there is no $u \in \partial U$ such that $u=v \mathcal{F} u$ for some $v \in(0,1)$. Consequently, by the nonlinear alternative of Leray-Schauder type, we deduce that $\mathcal{F}$ has a fixed point $u \in \bar{U}$ which is a solution of the boundary value problem (1.1). This completes the proof. 


\section{Examples}

In this section, we present some examples to illustrate our results.

Example 4.1 Consider the following fractional integral boundary value problem:

$$
\left\{\begin{array}{l}
{ }^{c} D^{\frac{3}{2}} u(t)=\frac{\sin ^{2}(2 \pi t)}{(3+t)^{3}} \cdot \frac{|u(t)|}{1+|u(t)|}+\frac{1}{2}, \quad 0<t<2, \\
\sqrt{2} I^{3 / 2} u(2)-\frac{1}{2} I^{\sqrt{3}} u(2)=1, \\
3 I^{\pi / 3} u(2 / 3)-\frac{7}{4} I^{\sqrt{2} / 2} u(4 / 3)=\frac{1}{2}\left(I^{\sqrt{\pi}} u(2)-I^{\sqrt{\pi}} u(5 / 3)\right)
\end{array}\right.
$$

Here $q=3 / 2, T=2, m=2, n=2, l=1, \omega=1, \alpha_{1}=3 / 2, \alpha_{2}=\sqrt{3}, \beta_{1}=\pi / 3, \beta_{2}=\sqrt{2} / 2$, $\gamma_{1}=\sqrt{\pi}, \theta_{1}=\sqrt{2}, \theta_{2}=-1 / 2, \lambda_{1}=3, \lambda_{2}=-7 / 4, \mu_{1}=1 / 2, \eta_{1}=2 / 3, \eta_{2}=4 / 3, \xi_{1}=5 / 3$, and $f(t, u)=\left(\sin ^{2}(2 \pi t)|u|\right) /\left((3+t)^{3}(1+|u|)\right)+(1 / 2)$. Since $|f(t, u)-f(t, v)| \leq(1 / 27)|u-v|,\left(\mathrm{H}_{1}\right)$ is satisfied with $L=1 / 27$. We can show that

$$
\begin{aligned}
\Lambda= & \frac{T^{q}}{\Gamma(q+1)}+\frac{\left(\left|\Omega_{3}\right|+\left|\Omega_{5}\right|\right)+\left(\left|\Omega_{6}\right|+\left|\Omega_{4}\right|\right) T}{|\Delta|} \sum_{i=1}^{m}\left|\theta_{i}\right| \frac{T^{\alpha_{i}+q}}{\Gamma\left(\alpha_{i}+q+1\right)} \\
& +\frac{\left|\Omega_{1}\right|+\left|\Omega_{2}\right| T}{|\Delta|}\left(\sum_{j=1}^{n}\left|\lambda_{j}\right| \frac{\eta_{j}^{\beta_{j}+q}}{\Gamma\left(\beta_{j}+q+1\right)}+\sum_{k=1}^{l}\left|\mu_{k}\right|\left(\frac{T^{\gamma_{k}+q}+\xi_{k}^{\gamma_{k}+q}}{\Gamma\left(\gamma_{k}+q+1\right)}\right)\right) \\
\approx & 14.13402398 .
\end{aligned}
$$

Thus $L \Lambda \approx 0.52348237<1$. Hence, by Theorem 3.1, the boundary value problem $(4.1)$ has a unique solution on $[0,2]$.

Example 4.2 Consider the following fractional integral boundary value problem:

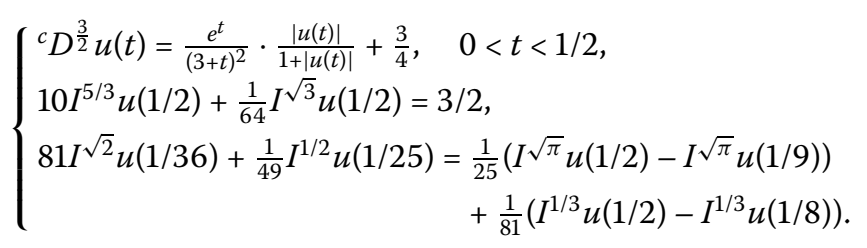

Here $q=3 / 2, T=1 / 2, m=2, n=2, l=2, \omega=3 / 2, \alpha_{1}=5 / 3, \alpha_{2}=\sqrt{3}, \beta_{1}=\sqrt{2}, \beta_{2}=1 / 2$, $\gamma_{1}=\sqrt{\pi}, \gamma_{2}=1 / 3, \theta_{1}=10, \theta_{2}=1 / 64, \lambda_{1}=81, \lambda_{2}=1 / 49, \mu_{1}=1 / 25, \mu_{2}=1 / 81, \eta_{1}=1 / 36, \eta_{2}=$ $1 / 25, \xi_{1}=1 / 9, \xi_{2}=1 / 8$, and $f(t, u)=\left(e^{t}|u|\right) /\left((3+t)^{2}(1+|u|)\right)+(3 / 4)$. We choose $h(t)=e^{t} / 9$ and we obtain

$$
\begin{aligned}
H^{*}= & I^{q} h(T)+\frac{\left(\left|\Omega_{5}\right|+\left|\Omega_{3}\right|\right)+\left(\left|\Omega_{6}\right|+\left|\Omega_{4}\right|\right) T}{|\Delta|} \sum_{i=1}^{m}\left|\theta_{i}\right| I^{\alpha_{i}+q} h(T) \\
& +\frac{\left|\Omega_{1}\right|+\left|\Omega_{2}\right| T}{|\Delta|}\left(\sum_{j=1}^{n}\left|\lambda_{j}\right| I^{\beta_{j}+q} h\left(\eta_{j}\right)+\sum_{k=1}^{l}\left|\mu_{k}\right|\left(I^{\gamma_{k}+q} h(T)+I^{\gamma_{k}+q} h\left(\xi_{k}\right)\right)\right) \\
\approx & 0.89374610 .
\end{aligned}
$$

Clearly,

$$
|f(t, u)-f(t, v)|=\frac{e^{t}}{(3+t)^{2}}\left(\frac{|u|-|v|}{1+|u|+|v|+|u||v|}\right) \leq \frac{e^{t}}{9}\left(\frac{|u-v|}{0.89374610+|u-v|}\right) .
$$


Hence, by Theorem 3.3, the boundary value problem (4.2) has a unique solution on $[0,1 / 2]$.

Example 4.3 Consider the following fractional integral boundary value problem:

$$
\left\{\begin{aligned}
{ }^{c} D^{\frac{3}{2}} u(t)=\frac{e^{-t} \sin ^{2}(2 \pi t)}{(2+t)^{2}} \cdot \frac{|u(t)|}{1+|u(t)|}+3 t, & 0<t<1, \\
256 I^{3 / 2} u(1)+\frac{1}{100} I^{\sqrt{3}} u(1)=1, & \\
1,024 I^{\pi / 3} u(1 / 5)+\frac{1}{1,024} I^{1 / \sqrt{2}} u(1 / 3)= & \frac{1}{512}\left(I^{\sqrt{\pi}} u(1)-I^{\sqrt{\pi}} u(1 / 3)\right) \\
& +\frac{1}{1,024}\left(I^{\sqrt{\pi} / 2} u(1)-I^{\sqrt{\pi} / 2} u(1 / 6)\right) .
\end{aligned}\right.
$$

Here $q=3 / 2, T=1, m=2, n=2, l=2, \omega=1, \alpha_{1}=3 / 2, \alpha_{2}=\sqrt{3}, \beta_{1}=\pi / 3, \beta_{2}=1 / \sqrt{2}, \gamma_{1}=$ $\sqrt{\pi}, \gamma_{2}=\sqrt{\pi} / 2, \theta_{1}=256, \theta_{2}=1 / 100, \lambda_{1}=1,024, \lambda_{2}=1 / 1,024, \mu_{1}=1 / 512, \mu_{2}=1 / 1,024$, $\eta_{1}=1 / 5, \eta_{2}=1 / 3, \xi_{1}=1 / 3, \xi_{2}=1 / 6$, and $f(t, u)=\left(e^{-t} \sin ^{2}(2 \pi t)|u|\right) /\left((2+t)^{2}(1+|u|)\right)+3 t$. Since $|f(t, u)-f(t, v)| \leq(1 / 4)|u-v|,\left(\mathrm{H}_{1}\right)$ is satisfied with $L=1 / 4$. We find that

$$
\begin{aligned}
& \frac{\left(\left|\Omega_{3}\right|+\left|\Omega_{5}\right|\right)+\left(\left|\Omega_{6}\right|+\left|\Omega_{4}\right|\right) T}{|\Delta|} \sum_{i=1}^{m}\left|\theta_{i}\right| \frac{T^{\alpha_{i}+q}}{\Gamma\left(\alpha_{i}+q+1\right)} \\
& +\frac{\left|\Omega_{1}\right|+\left|\Omega_{2}\right| T}{|\Delta|}\left(\sum_{j=1}^{n}\left|\lambda_{j}\right| \frac{\eta_{j}^{\beta_{j}+q}}{\Gamma\left(\beta_{j}+q+1\right)}+\sum_{k=1}^{l}\left|\mu_{k}\right|\left(\frac{T^{\gamma_{k}+q}+\xi_{k}^{\gamma_{k}+q}}{\Gamma\left(\gamma_{k}+q+1\right)}\right)\right) \\
& \approx 0.92525429<1 .
\end{aligned}
$$

Clearly,

$$
|f(t, u)|=\left|\frac{e^{-t} \sin ^{2}(2 \pi t)}{(2+t)^{2}} \cdot \frac{|u(t)|}{1+|u(t)|}+3 t\right| \leq \frac{e^{-t}}{4}+3 t .
$$

Hence, by Theorem 3.4, the boundary value problem (4.3) has at least one solution on $[0,1]$.

Example 4.4 Consider the following fractional integral boundary value problem:

$$
\left\{\begin{array}{l}
{ }^{c} D^{\frac{5}{3}} u(t)=\frac{1}{225}\left(1+t^{2}\right)\left(\frac{u^{2}}{1+|u|}+\frac{|u|}{1+|u|}\right), \quad 0<t<3, \\
I^{1 / 2} u(3)-\sqrt{3} I^{3 / 2} u(3)-\frac{4}{5} I^{\sqrt{2}} u(3)=\pi, \\
\frac{3}{2} I^{\sqrt{3}} u(2 / 5)=\frac{2}{9}\left(I^{8 / 5} u(3)-I^{8 / 5} u(3 / 4)\right)-10\left(I^{1 / 4} u(3)-I^{1 / 4} u(1 / 5)\right) .
\end{array}\right.
$$

Here $q=5 / 3, T=3, m=3, n=1, l=2, \omega=\pi, \alpha_{1}=1 / 2, \alpha_{2}=3 / 2, \alpha_{3}=\sqrt{2}, \beta_{1}=\sqrt{3}, \gamma_{1}=$ $8 / 5, \gamma_{2}=1 / 4, \theta_{1}=1, \theta_{2}=-\sqrt{3}, \theta_{3}=-4 / 5, \lambda_{1}=3 / 2, \mu_{1}=2 / 9, \mu_{2}=-10, \eta_{1}=2 / 5, \xi_{1}=3 / 4$, $\xi_{2}=1 / 5$, and $f(t, u)=(1 / 225)\left(1+t^{2}\right)\left(\left(u^{2} /(1+|u|)\right)+(|u| /(1+|u|))\right)$. Then we get

$$
\begin{aligned}
\Lambda= & \frac{T^{q}}{\Gamma(q+1)}+\frac{\left(\left|\Omega_{3}\right|+\left|\Omega_{5}\right|\right)+\left(\left|\Omega_{6}\right|+\left|\Omega_{4}\right|\right) T}{|\Delta|} \sum_{i=1}^{m}\left|\theta_{i}\right| \frac{T^{\alpha_{i}+q}}{\Gamma\left(\alpha_{i}+q+1\right)} \\
& +\frac{\left|\Omega_{1}\right|+\left|\Omega_{2}\right| T}{|\Delta|}\left(\sum_{j=1}^{n}\left|\lambda_{j}\right| \frac{\eta_{j}^{\beta_{j}+q}}{\Gamma\left(\beta_{j}+q+1\right)}+\sum_{k=1}^{l}\left|\mu_{k}\right|\left(\frac{T^{\gamma_{k}+q}+\xi_{k}^{\gamma_{k}+q}}{\Gamma\left(\gamma_{k}+q+1\right)}\right)\right)
\end{aligned}
$$


and

$$
\Phi=\frac{\left(\left|\Omega_{3}\right|+\left|\Omega_{5}\right|\right)+\left(\left|\Omega_{6}\right|+\left|\Omega_{4}\right|\right) T}{|\Delta|}\left|\omega_{1}\right| \approx 0.80515429
$$

Clearly,

$$
|f(t, u)|=\left|\frac{1}{225}\left(1+t^{2}\right)\left(\frac{u^{2}}{1+|u|}+\frac{|u|}{1+|u|}\right)\right| \leq \frac{1}{225}\left(1+t^{2}\right)(|u|+1) .
$$

Choosing $p(t)=(1 / 225)\left(1+t^{2}\right)$ and $\psi(|u|)=|u|+1$, we can show that

$$
\frac{M}{\psi(M)\|p\| \Lambda+\Phi}>1
$$

which implies that $M>4.55994347$. Hence, by Theorem 3.6, the boundary value problem (4.4) has at least one solution on $[0,3]$.

\section{Competing interests}

The authors declare that they have no competing interests.

\section{Authors' contributions}

All authors contributed equally in this article. They read and approved the final manuscript.

\section{Author details}

${ }^{1}$ Nonlinear Dynamic Analysis Research Center, Department of Mathematics, Faculty of Applied Science, King Mongkut's University of Technology North Bangkok, Bangkok, Thailand. '2Department of Mathematics, University of loannina, loannina, 451 10, Greece.

\section{Authors' information}

Sotiris K Ntouyas is a member of Nonlinear Analysis and Applied Mathematics (NAAM) Research Group at King Abdulaziz University, Jeddah, Saudi Arabia.

\section{Acknowledgements}

The research of J Tariboon is supported by King Mongkut's University of Technology North Bangkok, Thailand. This research of W Sudsutad is supported by the Centre of Excellence in Mathematics, the Commission on Higher Education, Thailand.

Received: 8 April 2014 Accepted: 25 June 2014 Published: 22 July 2014

\section{References}

1. Samko, SG, Kilbas, AA, Marichev, Ol: Fractional Integrals and Derivatives: Theory and Applications. Gordon \& Breach, Yverdon (1993)

2. Podlubny, I: Fractional Differential Equations. Academic Press, San Diego (1999)

3. Kilbas, AA, Srivastava, HM, Trujillo, JJ: Theory and Applications of Fractional Differential Equations. North-Holland Mathematics Studies, vol. 204. Elsevier, Amsterdam (2006)

4. Sabatier, J, Agrawal, OP, Machado, JAT (eds.): Advances in Fractional Calculus: Theoretical Developments and Applications in Physics and Engineering. Springer, Dordrecht (2007)

5. Lakshmikantham, V, Leela, S, Vasundhara Devi, J: Theory of Fractional Dynamic Systems. Cambridge Academic Publishers, Cambridge (2009)

6. Ahmad, B, Ntouyas, SK: Nonlinear fractional differential equations and inclusions of arbitrary order and multi-strip boundary conditions. Electron. J. Differ. Equ. 2012, 98 (2012)

7. Nyamoradi, $\mathrm{N}$, Javidi, M: Existence of multiple positive solutions for fractional differential inclusion with $m$-point boundary conditions and two fractional orders. Electron. J. Differ. Equ. 2012, 187 (2012)

8. Ahmad, B, Nieto, JJ: Sequential fractional differential equations with three-point boundary conditions. Comput. Math. Appl. 64, 3046-3052 (2012)

9. Ubriaco, MR: Entropies based on fractional calculus. Phys. Lett. A 373, 2516-2519 (2009)

10. Hamani, S, Benchohra, M, Graef, JR: Existence results for boundary-value problems with nonlinear fractional differential inclusions and integral conditions. Electron. J. Differ. Equ. 2010, 20 (2010)

11. Sudsutad, W, Tariboon, J: Existence results of fractional integro-differential equations with $m$-point multi-term fractional order integral boundary conditions. Bound. Value Probl. 2012, 94 (2012)

12. Sudsutad, W, Tariboon, J: Boundary value problems for fractional differential equations with three-point fractiona integral boundary conditions. Adv. Differ. Equ. 2012, 93 (2012) 
13. Ntouyas, SK: Existence results for nonlocal boundary value problems for fractional differential equations and inclusions with fractional integral boundary conditions. Discuss. Math., Differ. Incl. Control Optim. 33, 17-39 (2013)

14. Ntouyas, SK: Boundary value problems for nonlinear fractional differential equations and inclusions with nonlocal and fractional integral boundary conditions. Opusc. Math. 33, 117-138 (2013)

15. Guezane-Lakoud, A, Khaldi, R: Solvability of a fractional boundary value problem with fractional integral condition. Nonlinear Anal. 75, 2692-2700 (2012)

16. Ahmad, B, Ntouyas, SK, Assolani, A: Caputo type fractional differential equations with nonlocal Riemann-Liouville integral boundary conditions. J. Appl. Math. Comput. 41, 339-350 (2013)

17. Liu, XY, Liu, YL: Fractional differential equations with fractional non-separated boundary conditions. Electron. J. Differ. Equ. 2013, 25 (2013)

18. Liu, XY, Liu, ZH, Fu, X: Relaxation in nonconvex optimal control problems described by fractional differential equations. J. Math. Anal. Appl. 409, 446-458 (2014)

19. Alsaedi, A, Ntouyas, SK, Agarwal, RP, Ahmad, B: A nonlocal multi-point multi-term fractional boundary value problem with Riemann-Liouville type integral boundary conditions involving two indices. Adv. Differ. Equ. 2013, 369 (2013)

20. Ahmad, B, Ntouyas, SK, Alsaedi, A: A study of nonlinear fractional differential equations of arbitrary order with Riemann-Liouville type multi-strip boundary conditions. Math. Probl. Eng. 2013, Article ID 320415 (2013)

21. Alsaedi, A, Ntouyas, SK, Ahmad, B: Existence results for Langevin fractional differential inclusions involving two fractional orders with four-point multi-term fractional integral boundary conditions. Abstr. Appl. Anal. 2013, Article ID 869837 (2013)

22. Boyd, DW, Wong, JSW: On nonlinear contractions. Proc. Am. Math. Soc. 20, 458-464 (1969)

23. Krasnoselskii, MA: Two remarks on the method of successive approximations. Usp. Mat. Nauk 10, $123-127$ (1955)

24. Granas, A, Dugundji, J: Fixed Point Theory. Springer, New York (2003)

doi:10.1186/1687-1847-2014-181

Cite this article as: Tariboon et al.: Fractional integral problems for fractional differential equations via Caputo

derivative. Advances in Difference Equations 2014 2014:181.

\section{Submit your manuscript to a SpringerOpen ${ }^{\circ}$ journal and benefit from:}

- Convenient online submission

- Rigorous peer review

- Immediate publication on acceptance

- Open access: articles freely available online

- High visibility within the field

- Retaining the copyright to your article 